\title{
Mapping Workspaces to Virtual Space in Work Using Heterogeneous Haptic Interface Devices
}

\author{
Ayano Tatematsu and Yutaka Ishibashi \\ Nagoya Institute of Technology \\ Japan
}

\section{Introduction}

In networked haptic environments, users can touch objects in a virtual space and feel the weight of the objects by manipulating haptic interface devices (Srinivasan \& Basdogn, 1997). Thus, we can largely improve the efficiency of collaborative work such as remote surgery simulation and immerse ourselves in playing networked games.

On the other hand, a variety of haptic interface devices have been developed so far. The haptic interface devices have different specifications (e.g., the workspace size, position resolution, and exertable force) from each other. When we interconnect the devices over a network, the differences may cause some problems. There are a few papers addressing the problems (Hirose et al., 1998; Kameyama \& Ishibashi, 2007; Fujimoto et al., 2008; Huang et al., 2008). In (Hirose et al., 1998), Hirose et al. develop basic software called Haptic Interface Platform (HIP), which does not depend on types of haptic interface devices. Then, they show that users do not notice meaningful differences in hardness in an experiment where the users recognize the hardness of an object although the users manipulate different types of haptic interface devices. In (Kameyama \& Ishibashi, 2007), the authors clarify the influences of difference in workspace size between PHANToM Omni (Salisbury \& Srinivasan, 1997) (just called Omni here) and PHANToM Desktop (Salisbury \& Srinivasan, 1997) (called Desktop) for networked collaborative work and competitive work. They show that if the range of motion of a haptic interface device is not limited to a workspace which is smaller than the virtual space, there is no large influence of the difference on the efficiency of the collaborative work and the fairness of the competitive work. Otherwise, the efficiency of the collaborative work seriously deteriorates, and the fairness is damaged in the competitive work. In (Fujimoto et al., 2008), the authors handle collaborative work using Omni and SPIDAR-G AHS (Kim et al., 2003) (called SPIDAR). And they compare some methods of mapping workspaces to a virtual space. In (Huang et al., 2008), the authors treat collaborative work using Omni, Desktop, SPIDAR, and Falcon (Novint, 2007) when the size of a virtual space is small so that it is not necessary to map workspaces to the virtual space. However, the experiment with various haptic interface devices in the case where we need mapping (that is, the size of a virtual space is different from the size of each workspace) has not been performed. 
In this chapter, we deal with collaborative work and competitive work using Omni, Desktop, SPIDAR, and Falcon. And we examine the influences of methods of mapping workspaces to a virtual space on the efficiency of the two types of work.

The rest of this chapter is organized as follows. Section 2 outlines the specifications of the haptic interface devices. Section 3 gives a brief description of the collaborative work and the competitive work. Section 4 explains system models of the two types of work. Section 5 describes methods of mapping. Section 6 explains the method of our experiment, and experimental results are presented in Section 7 . Section 8 concludes the chapter.

\section{Specifications of Haptic Interface Devices}

When a user uses Omni or Desktop (see Figures 1(a) and (b)), the user manipulates the stylus of the device as if he/she had a pen. When he/she employs SPIDAR (see Figure 1(c)), he/she manipulates a globe (called the grip) hung with eight wires. In the case of Falcon (see Figure $1(\mathrm{~d})$ ), he/she manipulates a spherical grip connected with three arms. The workspace sizes of the devices are different from each other (see Table 1). In addition, the position resolution and exertable force of each device are different from those of the other devices.

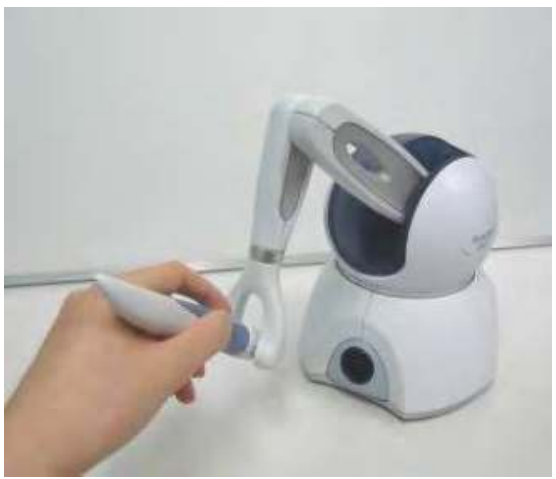

(a) Omni

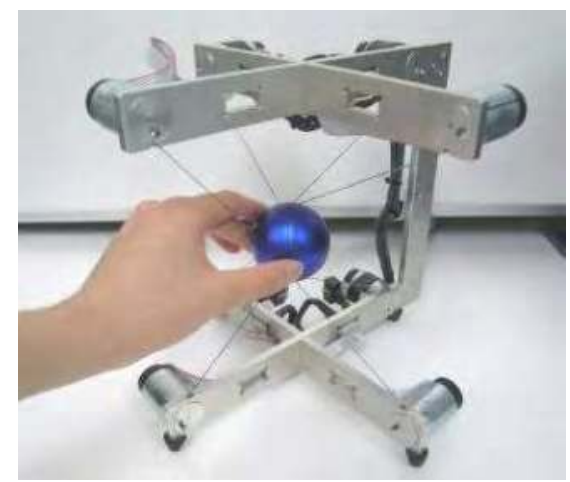

(c) SPIDAR

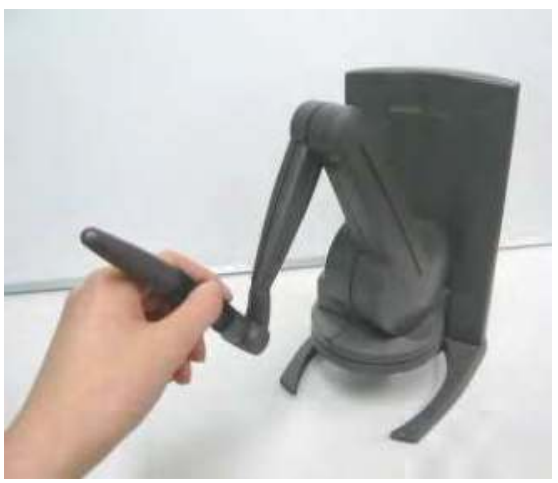

(b) Desktop

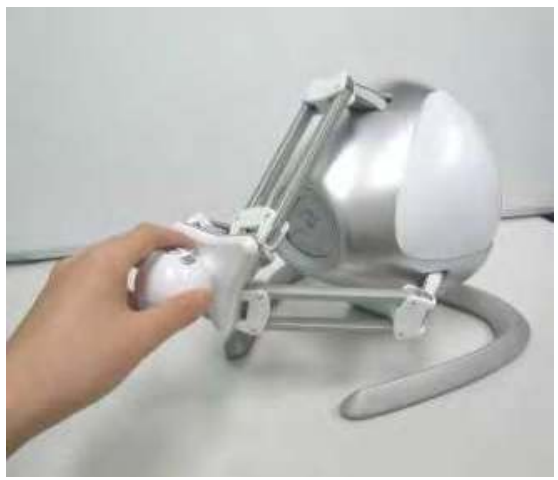

(d) Falcon

Fig. 1. Haptic interface devices 


\begin{tabular}{|l|r|r|r|}
\hline Device & Width [mm] & Height [mm] & Depth [mm] \\
\hline Omni & 160 & 120 & 70 \\
\hline Desktop & 160 & 120 & 120 \\
\hline SPIDAR & 200 & 120 & 200 \\
\hline Falcon & 75 & 75 & 75 \\
\hline
\end{tabular}

Table 1. Workspace sizes of haptic interface devices

\section{Work Descriptions}

We handle two types of work in which the difference in specifications excluding the workspace size among the four devices does not largely affect the efficiency of work.

\subsection{Collaborative Work}

Each of two users operates a haptic interface device, and the two users move a rigid cube (the length of each side is $30 \mathrm{~mm}$, and the mass is $800 \mathrm{~g}$ ) as an object collaboratively by holding the cube between the two cursors of the devices in a 3-D virtual space (width: 150 $\mathrm{mm}$, height: $150 \mathrm{~mm}$, depth: $140 \mathrm{~mm}$. We will discuss the size of the virtual space in Section 5) surrounded by walls, a floor, and a ceiling (see Figure 2) (Fujimoto et al., 2008; Huang et al., 2008). The cursor of each haptic interface device moves in the virtual space when a user manipulates the stylus or grip of the device with his/her hand. The two users lift and move the cube collaboratively so that the cube contains a target (a sphere in Figure 2) which revolves along a circular orbit at a constant velocity. We do not carry out collision detection among the target, the orbit, and the cube or cursors.

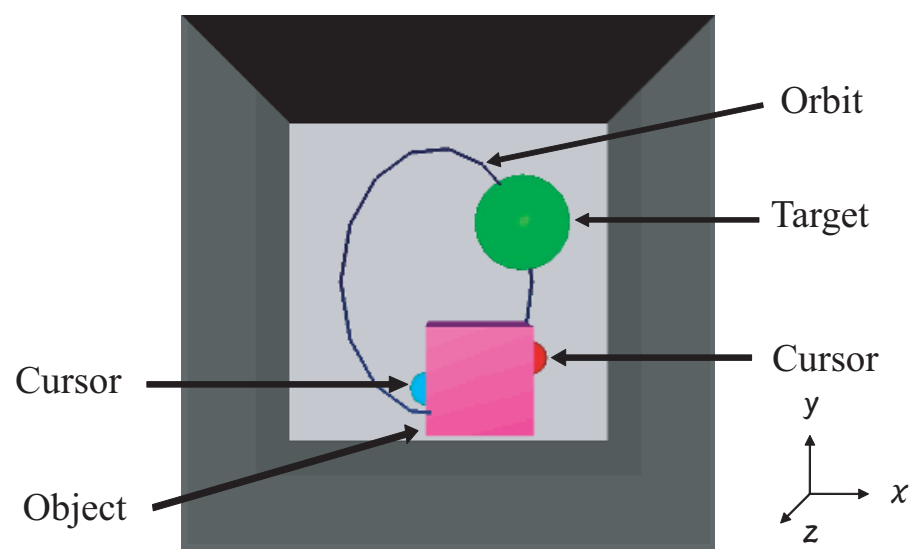

Fig. 2. Displayed image of virtual space in collaborative work

\subsection{Competitive Work}

Each of four players moves his/her object by lifting the object (the length of each side is 20 $\mathrm{mm}$, and the mass is $750 \mathrm{~g}$ ) from the bottom so that the object contains the target in a 3-D virtual space (width: $150 \mathrm{~mm}$, height: $150 \mathrm{~mm}$, depth: $140 \mathrm{~mm}$. We will discuss the size of the virtual space in Section 5) as shown in Figure 3. If the distance between the center of the object and that of the target is less than $5 \mathrm{~mm}$, we judge that the object contains the target. 
When the target is contained by any of the four objects, it disappears and then appears at a randomly-selected position in the space. The four players compete on the number of eliminated targets with each other. The objects and target do not collide with each other, and the cursors do not collide with the target.

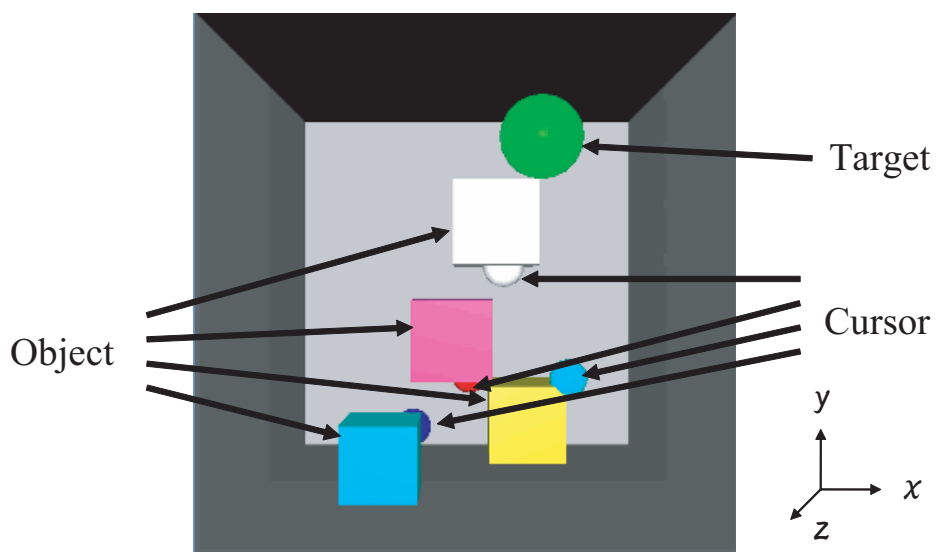

Fig. 3. Displayed image of virtual space in competitive work

\section{System Models}

\subsection{Collaborative Work}

A system model of the collaborative work is shown in Figure 4. The system model is based on a client-server model which consists of a single server and two clients (clients 1 and 2). As a haptic interface device, we employ Omni, Desktop, SPIDAR, or Falcon.

When the haptic interface device at a client is Omni, Desktop, or Falcon, the client performs haptic simulation by repeating the servo loop at a rate of $1 \mathrm{kHz}$ (Novint, 2007; SensAble, 2004). And it inputs/outputs a stream of media units (MUs), each of which is the information unit for intra-stream synchronization, at the rate; that is, an MU is input/output every millisecond. Each MU contains the identification (ID) number of the client, the positional information of the cursor of the partner device, and the sequence number of the servo loop, which we use instead of the timestamp of the MU (Ishibashi et al., 2002). In the case where SPIDAR is used at a client, the client carries out haptic simulation at $1 \mathrm{kHz}$ by using a timer and inputs/outputs a stream of MUs in the same way as that in the case where the other haptic interface devices are employed.

The server receives MUs from the two clients, and it calculates the position of the object based on the spring-damper model (SensAble, 2004). Then, it transmits the positional information of the object and cursor as an MU to the two clients.

When each client receives an MU, the client updates the position of the object after carrying out intra-stream synchronization control and calculates the reaction force applied to a user of the client. We employ Skipping (Ishibashi et al., 2002) for the intra-stream synchronization control at the clients. Skipping outputs MUs on receiving the MUs. When multiple MUs are received at the same time, however, only the latest MU is output and the others are discarded. 


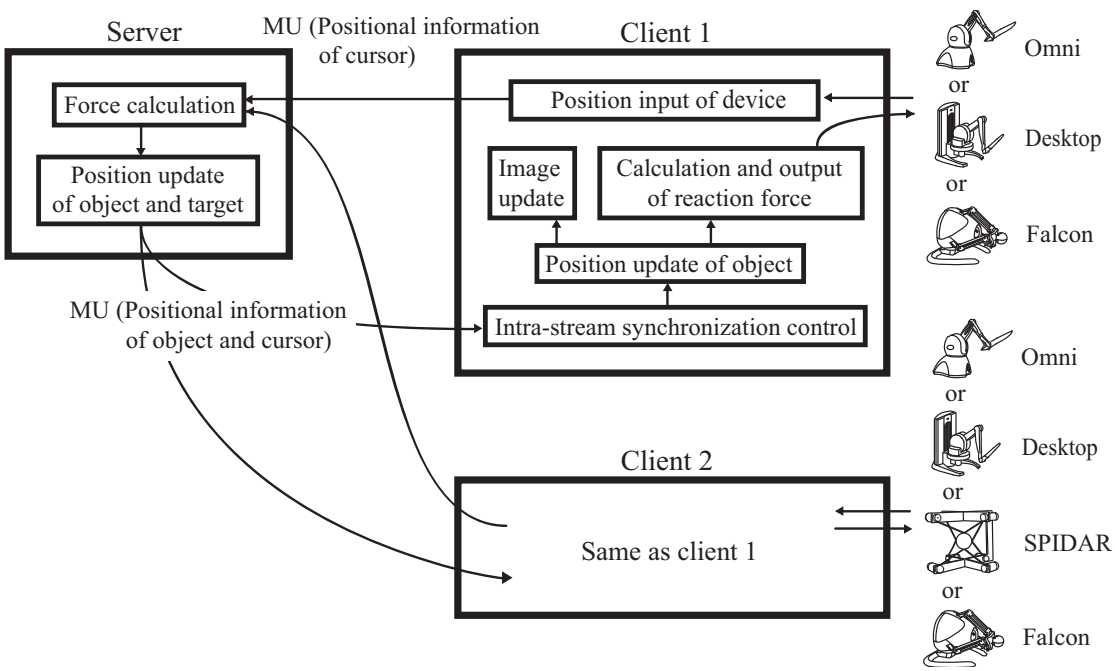

Fig. 4. System model of collaborative work

\subsection{Competitive Work}

Figure 5 shows a system model of the competitive work. The system model is similar to that of the collaborative work; that is, functions at the server and each client are almost the same as those of the collaborative work. The system model includes four clients (clients 1 through 4).

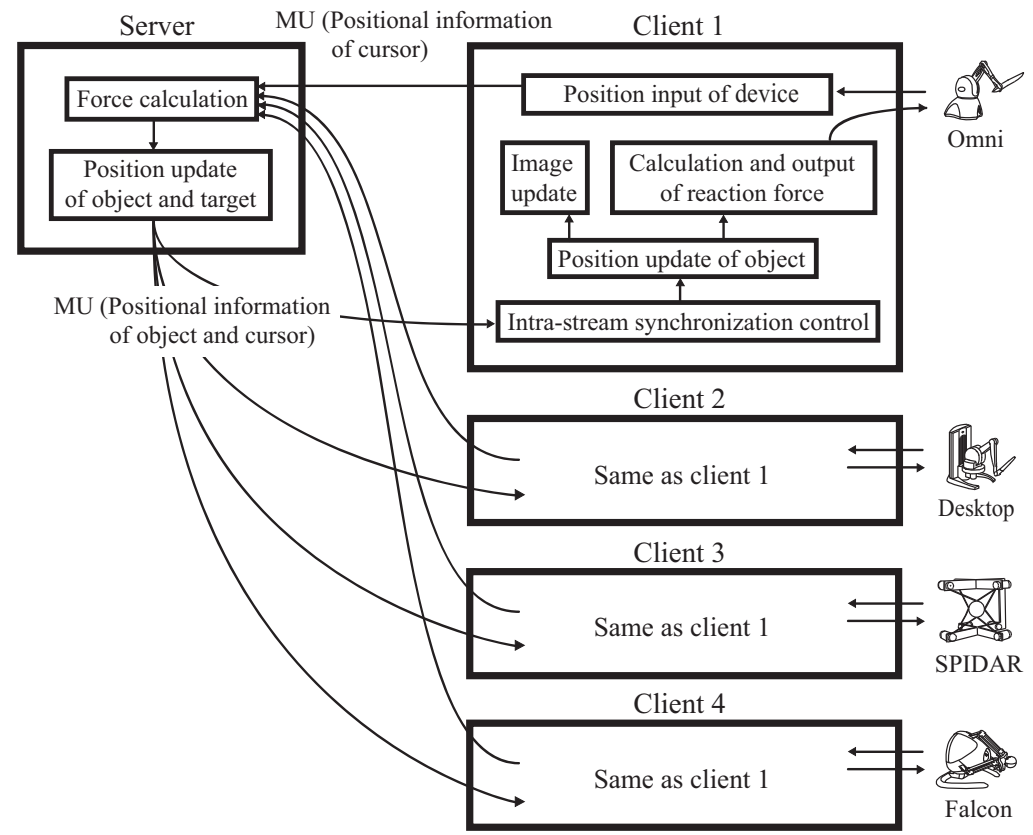

Fig. 5. System model of competitive work 


\section{Methods of Mapping}

When the size of the virtual space is different from that of each workspace, there may exist domains that some of the haptic interface devices cannot reach in the virtual space. Therefore, it is necessary to map the workspace to the virtual space so that each device is able to work throughout the virtual space.

In this chapter, we deal with four cases in terms of the virtual space size. For explanation of the four cases, we define the reference size (width: $75.0 \mathrm{~mm}$, height: $75.0 \mathrm{~mm}$, depth: $70.0 \mathrm{~mm}$ ) as the intersection of the four workspace sizes. In the first case, we set the virtual space size to half the reference size (width: $37.5 \mathrm{~mm}$, height: $37.5 \mathrm{~mm}$, depth: $35.0 \mathrm{~mm}$ ). In the second case, the virtual space size is set to the reference size. In the third case, the virtual space size is set to one and a half times the reference size (width: $112.5 \mathrm{~mm}$, height: $112.5 \mathrm{~mm}$, depth: $105 \mathrm{~mm}$ ). In the fourth case, the virtual space size is set to twice the reference size (width: $150 \mathrm{~mm}$, height: $150 \mathrm{~mm}$, depth: $140 \mathrm{~mm}$ ). However, in the collaborative work, the first case is not treated since it was difficult to do the work due to the relation between the size of the object (see Section 3. The size of the object is constant independently of the size of the virtual space) and that of the virtual space.

This chapter handles the following two methods of mapping a workspace to the virtual space.

Method $a$ : The workspace is uniformly mapped to the virtual space in the directions of the $x$-, $y$-, and $z$-axes (see Figure 6, which shows the shape of the workspace before and after mapping with Method a). In the case where the haptic interface device is Omni and the virtual space size is set to the reference size, for example, since the mapping ratio of the $z$ axis direction is one and the ratio is larger than those of the other axial directions, we also set the ratios of the other axial directions to one (see Tables 2, which show the mapping ratios in the two methods in the collaborative work in the case where the virtual space size is set to the reference size. We also show the mapping ratios in the collaborative work and competitive work in Tables 3 through 8).

Method $b$ : The workspace is individually mapped to the virtual space in the direction of each axis so that the mapped workspace size corresponds to the virtual space size (see Figure 7, which shows the shape of the workspace before and after mapping with Method $b$ ).

In addition, we handled other two methods. In one method, the mapping ratio of each employed device is set to the largest mapping ratio among the employed devices in Method a. In the other method, mapping ratio of each employed device is set to the largest mapping ratio among the employed devices in Method $b$. However, experimental results of the two methods were worse than those of Method a.

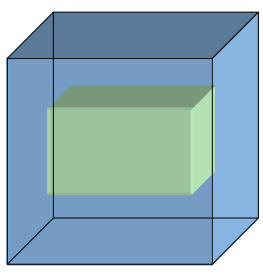

Before

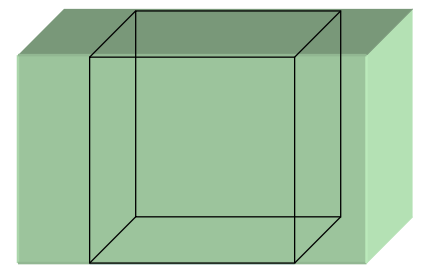

After

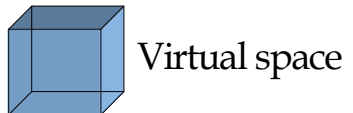

Workspace

Fig. 6. Illustration of mapping with Method a 


\begin{tabular}{|c|c|c|c|c|c|}
\hline Method & Combination & Device & $\begin{array}{c}\text { Ratio of } \\
x \text {-axis }\end{array}$ & $\begin{array}{c}\text { Ratio of } \\
y \text {-axis }\end{array}$ & $\begin{array}{c}\text { Ratio of } \\
z \text {-axis }\end{array}$ \\
\hline \multirow{9}{*}{$\mathrm{a}$} & Omni-Omni & Omni & 1.00 & 1.00 & 1.00 \\
\hline & Desktop-Desktop & Desktop & 0.63 & 0.63 & 0.63 \\
\hline & Falcon-Falcon & Falcon & 1.00 & 1.00 & 1.00 \\
\hline & \multirow{2}{*}{ Omni-Desktop } & Omni & 1.00 & 1.00 & 1.00 \\
\hline & & Desktop & 0.63 & 0.63 & 0.63 \\
\hline & \multirow{2}{*}{ Falcon-Omni } & Falcon & 1.00 & 1.00 & 1.00 \\
\hline & & Omni & 1.00 & 1.00 & 1.00 \\
\hline & \multirow{2}{*}{ Falcon-SPIDAR } & Falcon & 1.00 & 1.00 & 1.00 \\
\hline & & SPIDAR & 0.63 & 0.63 & 0.63 \\
\hline \multirow{9}{*}{$\mathrm{b}$} & Omni-Omni & Omni & 0.47 & 0.63 & 1.00 \\
\hline & Desktop-Desktop & Desktop & 0.47 & 0.63 & 0.58 \\
\hline & Falcon-Falcon & Falcon & 1.00 & 1.00 & 0.93 \\
\hline & \multirow{2}{*}{ Omni-Desktop } & Omni & 0.47 & 0.63 & 1.00 \\
\hline & & Desktop & 0.47 & 0.63 & 0.58 \\
\hline & \multirow{2}{*}{ Falcon-Omni } & Falcon & 1.00 & 1.00 & 0.93 \\
\hline & & Omni & 0.47 & 0.63 & 1.00 \\
\hline & \multirow{2}{*}{ Falcon-SPIDAR } & Falcon & 1.00 & 1.00 & 0.93 \\
\hline & & SPIDAR & 0.38 & 0.63 & 0.35 \\
\hline
\end{tabular}

Table 2. Mapping ratios in two methods of mapping in collaborative work in case where virtual space size is set to reference size

\begin{tabular}{|c|c|c|c|c|c|}
\hline Method & Combination & Device & $\begin{array}{c}\text { Ratio of } \\
x \text {-axis }\end{array}$ & $\begin{array}{c}\text { Ratio of } \\
y \text {-axis }\end{array}$ & $\begin{array}{c}\text { Ratio of } \\
z \text {-axis }\end{array}$ \\
\hline \multirow{9}{*}{ a } & Omni-Omni & Omni & 1.50 & 1.50 & 1.50 \\
\hline & Desktop-Desktop & Desktop & 0.94 & 0.94 & 0.94 \\
\hline & Falcon-Falcon & Falcon & 1.50 & 1.50 & 1.50 \\
\hline & \multirow{2}{*}{ Omni-Desktop } & Omni & 1.50 & 1.50 & 1.50 \\
\hline & & Desktop & 0.94 & 0.94 & 0.94 \\
\hline & \multirow{2}{*}{ Falcon-Omni } & Falcon & 1.50 & 1.50 & 1.50 \\
\hline & & Omni & 1.50 & 1.50 & 1.50 \\
\hline & \multirow{2}{*}{ Falcon-SPIDAR } & Falcon & 1.50 & 1.50 & 1.50 \\
\hline & & SPIDAR & 0.94 & 0.94 & 0.94 \\
\hline \multirow{9}{*}{ b } & Omni-Omni & Omni & 0.70 & 0.94 & 1.50 \\
\hline & Desktop-Desktop & Desktop & 0.70 & 0.94 & 0.88 \\
\hline & Falcon-Falcon & Falcon & 1.50 & 1.50 & 1.40 \\
\hline & \multirow{2}{*}{ Omni-Desktop } & Omni & 0.70 & 0.94 & 1.50 \\
\hline & & Desktop & 0.70 & 0.94 & 0.88 \\
\hline & \multirow{2}{*}{ Falcon-Omni } & Falcon & 1.50 & 1.50 & 1.40 \\
\hline & & Omni & 0.70 & 0.94 & 1.50 \\
\hline & \multirow{2}{*}{ Falcon-SPIDAR } & Falcon & 1.50 & 1.50 & 1.40 \\
\hline & & SPIDAR & 0.56 & 0.94 & 0.53 \\
\hline
\end{tabular}

Table 3. Mapping ratios in two methods of mapping in collaborative work in case where virtual space size is set to one and a half times reference size 


\begin{tabular}{|c|c|c|c|c|c|}
\hline Method & Combination & Device & $\begin{array}{c}\text { Ratio of } \\
x \text {-axis }\end{array}$ & $\begin{array}{c}\text { Ratio of } \\
y \text {-axis }\end{array}$ & $\begin{array}{c}\text { Ratio of } \\
z \text {-axis }\end{array}$ \\
\hline \multirow{9}{*}{ a } & Omni-Omni & Omni & 2.00 & 2.00 & 2.00 \\
\hline & Desktop-Desktop & Desktop & 1.25 & 1.25 & 1.25 \\
\hline & Falcon-Falcon & Falcon & 2.00 & 2.00 & 2.00 \\
\hline & \multirow{2}{*}{ Omni-Desktop } & Omni & 2.00 & 2.00 & 2.00 \\
\hline & & Desktop & 1.25 & 1.25 & 1.25 \\
\hline & \multirow{2}{*}{ Falcon-Omni } & Falcon & 2.00 & 2.00 & 2.00 \\
\hline & & Omni & 2.00 & 2.00 & 2.00 \\
\hline & \multirow{2}{*}{ Falcon-SPIDAR } & Falcon & 2.00 & 2.00 & 2.00 \\
\hline & & SPIDAR & 1.25 & 1.25 & 1.25 \\
\hline \multirow{9}{*}{ b } & Omni-Omni & Omni & 0.94 & 1.25 & 2.00 \\
\hline & Desktop-Desktop & Desktop & 0.94 & 1.25 & 1.17 \\
\hline & Falcon-Falcon & Falcon & 2.00 & 2.00 & 1.87 \\
\hline & \multirow{2}{*}{ Omni-Desktop } & Omni & 0.94 & 1.25 & 2.00 \\
\hline & & Desktop & 0.94 & 1.25 & 1.17 \\
\hline & \multirow{2}{*}{ Falcon-Omni } & Falcon & 2.00 & 2.00 & 1.87 \\
\hline & & Omni & 0.94 & 1.25 & 2.00 \\
\hline & \multirow{2}{*}{ Falcon-SPIDAR } & Falcon & 2.00 & 2.00 & 1.87 \\
\hline & & SPIDAR & 0.75 & 1.25 & 0.70 \\
\hline
\end{tabular}

Table 4. Mapping ratios in two methods of mapping in collaborative work

\begin{tabular}{|c|l|c|c|c|}
\hline Method & Device & $\begin{array}{c}\text { Ratio of } \\
x \text {-axis }\end{array}$ & $\begin{array}{c}\text { Ratio of } \\
y \text {-axis }\end{array}$ & $\begin{array}{c}\text { Ratio of } \\
z \text {-axis }\end{array}$ \\
\hline \multirow{4}{*}{ a } & Omni & 0.50 & 0.50 & 0.50 \\
\cline { 2 - 5 } & Desktop & 0.31 & 0.31 & 0.31 \\
\cline { 2 - 5 } & SPIDAR & 0.31 & 0.31 & 0.31 \\
\cline { 2 - 5 } & Falcon & 0.50 & 0.50 & 0.50 \\
\hline \multirow{4}{*}{$\mathrm{b}$} & Omni & 0.23 & 0.31 & 0.50 \\
\cline { 2 - 5 } & Desktop & 0.23 & 0.31 & 0.29 \\
\cline { 2 - 5 } & SPIDAR & 0.19 & 0.31 & 0.18 \\
\cline { 2 - 5 } & Falcon & 0.50 & 0.50 & 0.47 \\
\hline
\end{tabular}

Table 5. Mapping ratios in two methods of mapping in competitive work in case where virtual space size is set to half reference size

\begin{tabular}{|c|l|c|c|c|}
\hline Method & Device & $\begin{array}{c}\text { Ratio of } \\
x \text {-axis }\end{array}$ & $\begin{array}{c}\text { Ratio of } \\
y \text {-axis }\end{array}$ & $\begin{array}{c}\text { Ratio of } \\
z \text {-axis }\end{array}$ \\
\hline \multirow{3}{*}{ a } & Omni & 1.00 & 1.00 & 1.00 \\
\cline { 2 - 5 } & Desktop & 0.63 & 0.63 & 0.63 \\
\cline { 2 - 5 } & SPIDAR & 0.63 & 0.63 & 0.63 \\
\cline { 2 - 5 } & Falcon & 1.00 & 1.00 & 1.00 \\
\hline \multirow{3}{*}{$\mathrm{b}$} & Omni & 0.47 & 0.63 & 1.00 \\
\cline { 2 - 5 } & Desktop & 0.47 & 0.63 & 0.58 \\
\cline { 2 - 5 } & SPIDAR & 0.38 & 0.63 & 0.35 \\
\cline { 2 - 5 } & Falcon & 1.00 & 1.00 & 0.93 \\
\hline
\end{tabular}

Table 6. Mapping ratios in two methods of mapping in competitive work in case where virtual space size is set to reference size 


\begin{tabular}{|c|l|c|c|c|}
\hline Method & Device & $\begin{array}{c}\text { Ratio of } \\
x \text {-axis }\end{array}$ & $\begin{array}{c}\text { Ratio of } \\
y \text {-axis }\end{array}$ & $\begin{array}{c}\text { Ratio of } \\
z \text {-axis }\end{array}$ \\
\hline \multirow{4}{*}{ a } & Omni & 1.50 & 1.50 & 1.50 \\
\cline { 2 - 5 } & Desktop & 0.94 & 0.94 & 0.94 \\
\cline { 2 - 5 } & SPIDAR & 0.94 & 0.94 & 0.94 \\
\cline { 2 - 5 } & Falcon & 1.50 & 1.50 & 1.50 \\
\hline \multirow{4}{*}{$\mathrm{b}$} & Omni & 0.70 & 0.94 & 1.50 \\
\cline { 2 - 5 } & Desktop & 0.70 & 0.94 & 0.88 \\
\cline { 2 - 5 } & SPIDAR & 0.56 & 0.94 & 0.53 \\
\cline { 2 - 5 } & Falcon & 1.50 & 1.50 & 1.40 \\
\hline
\end{tabular}

Table 7. Mapping ratios in two methods of mapping in competitive work in case where virtual space size is set to one and a half times reference size

\begin{tabular}{|c|l|c|c|c|}
\hline Method & Device & $\begin{array}{c}\text { Ratio of } \\
x \text {-axis }\end{array}$ & $\begin{array}{c}\text { Ratio of } \\
y \text {-axis }\end{array}$ & $\begin{array}{c}\text { Ratio of } \\
z \text {-axis }\end{array}$ \\
\hline \multirow{4}{*}{ a } & Omni & 2.00 & 2.00 & 2.00 \\
\cline { 2 - 5 } & Desktop & 1.25 & 1.25 & 1.25 \\
\cline { 2 - 5 } & SPIDAR & 1.25 & 1.25 & 1.25 \\
\cline { 2 - 5 } & Falcon & 2.00 & 2.00 & 2.00 \\
\hline \multirow{4}{*}{$\mathrm{b}$} & Omni & 0.94 & 1.25 & 2.00 \\
\cline { 2 - 5 } & Desktop & 0.94 & 1.25 & 1.17 \\
\cline { 2 - 5 } & SPIDAR & 0.75 & 1.25 & 0.70 \\
\cline { 2 - 5 } & Falcon & 2.00 & 2.00 & 1.87 \\
\hline
\end{tabular}

Table 8. Mapping ratios in two methods of mapping in competitive work in case where virtual space size is set to twice reference size

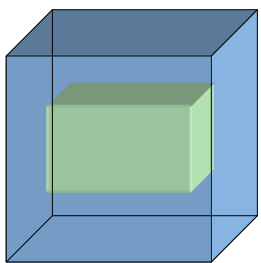

Before

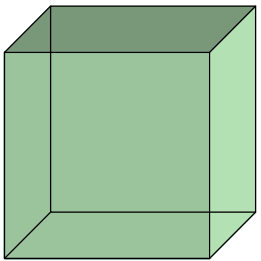

After

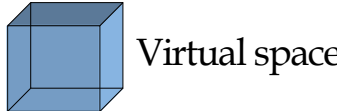

Workspace

Fig. 7. Illustration of mapping with Method b

\section{Method of Experiment}

\subsection{Experimental Systems}

As shown in Figure 8, our experimental system in the collaborative work consists of a single server and two clients (clients 1 and 2). The server is connected to the two clients via an Ethernet switching hub (100 Mbps). In this chapter, we deal with the following six combinations as pairs of the devices: Omni-Omni, Desktop-Desktop, Falcon-Falcon, OmniDesktop, Falcon-Omni, and Falcon-SPIDAR. These combinations are chosen from among pairs which have large differences in the efficiency of the work in (Huang et al., 2008). 


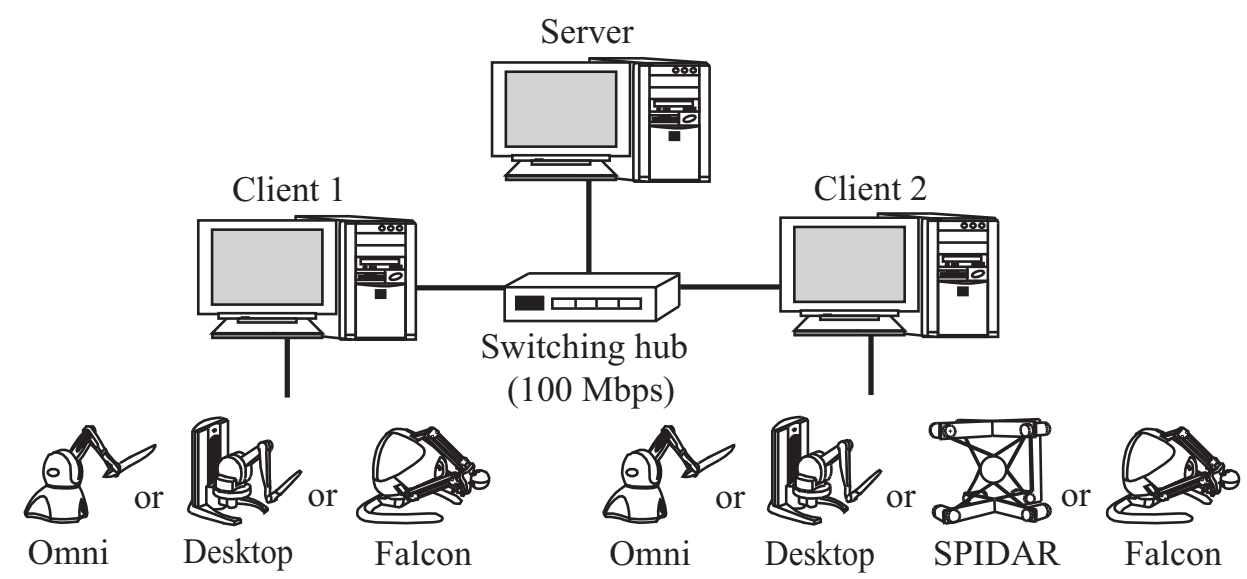

Fig. 8. Configuration of experimental system in collaborative work

Figure 9 shows our experimental system in the competitive work. The system consists of a single server and four clients (clients 1, 2, 3 and 4). The server is connected to the four clients via an Ethernet switching hub (100 Mbps). Clients 1 through 4 have Omni, Desktop, SPIDAR and Falcon, respectively.

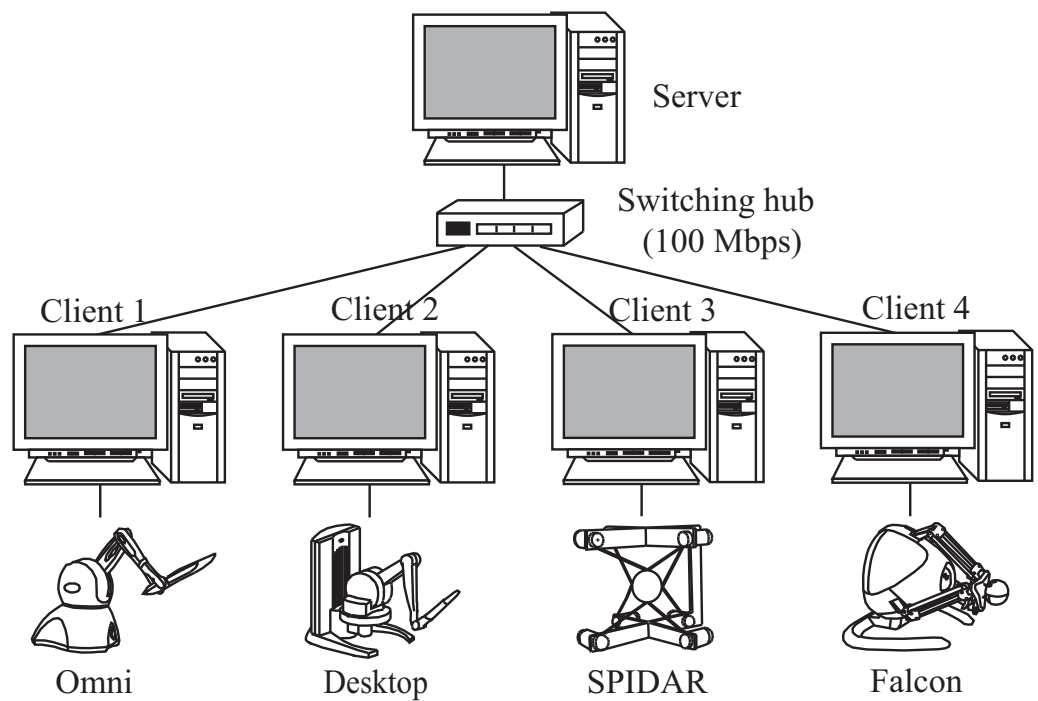

Fig. 9. Configuration of experimental system in competitive work

\subsection{Performance Measure}

As a performance measure, we employ the average distance between cube and target (Ishibashi et al., 2002) in the experiment on the collaborative work and the average total number of eliminated targets (Ishibashi \& Kaneoka, 2006) in the experiment on the competitive work, 
which are QoS (Quality of Service) parameters. The average distance between cube and target is defined as the mean distance between the centers of them. This measure is related to the accuracy of the collaborative work. Small values of the average distance indicate that the cube follows the target precisely; this signifies that the efficiency of the work is high. The average total number of eliminated targets is closely related to the efficiency of the competitive work. Large values lead to high efficiency of the work.

In the collaborative work, two users operated haptic interface devices at clients 1 and 2. The experiment for each method was carried out 40 times. When the users operated different devices from each other, they exchanged the devices, and the experiment was done again. In the competitive work, four users operated devices at clients 1, 2, 3 and 4 . The experiment for each method was also carried out 40 times. The users exchanged the devices every 10 times so that each user employed every device. The measurement time of each experimental run was 30 seconds in the two types of work.

\section{Experimental Results}

\subsection{Collaborative Work}

We show the average distance between cube and target for the two methods in Figures 10 through 12, where the virtual space size is set to the reference size, one and a half times the reference size, and twice the reference size, respectively. In the figures, we also display the $95 \%$ confidence intervals.

In Figures 10 through 12, we see that as the size of the virtual space becomes larger, the average distance increases. From this, we can say that the larger the size of the virtual space, the more difficult the work.

From Figures 10 through 12, we also find that the average distance of Method a is smaller than that of Method b in all the combinations. The reason is as follows. In Method b, the movement distances of the cursor in the directions of the three axes are different from each other in the virtual space even if the movement distances of the stylus or grip in the directions of the three axes are the same in the workspace. Thus, the work with Method $b$ is more difficult than that with Method a. In the case of Falcon-Falcon, the average distance of Method a is approximately equal to that of Method b. This is because the shape of the workspace of Falcon resembles that of the virtual space (the width, height, and depth of the workspace of Falcon are $75 \mathrm{~mm}$, and those of the virtual space are $75 \mathrm{~mm}, 75 \mathrm{~mm}$, and 70 $\mathrm{mm}$, respectively, in the case where the virtual space size is set to the reference size).

From the above observations, we can conclude that Method a is more effective than Method $\mathrm{b}$ in the collaborative work.

\subsection{Competitive Work}

We show the average total number of eliminated targets for the two methods in Figures 13 through 16, where the virtual space size is set to half the reference size, the reference size, one and a half times the reference size, and twice the reference size, respectively. In the figures, we also display the $95 \%$ confidence intervals.

In Figures 13 through 16, we see that as the size of the virtual space becomes larger, the average total number of eliminated targets decreases. From this, we can say that the larger the size of the virtual space, the more difficult the work. 


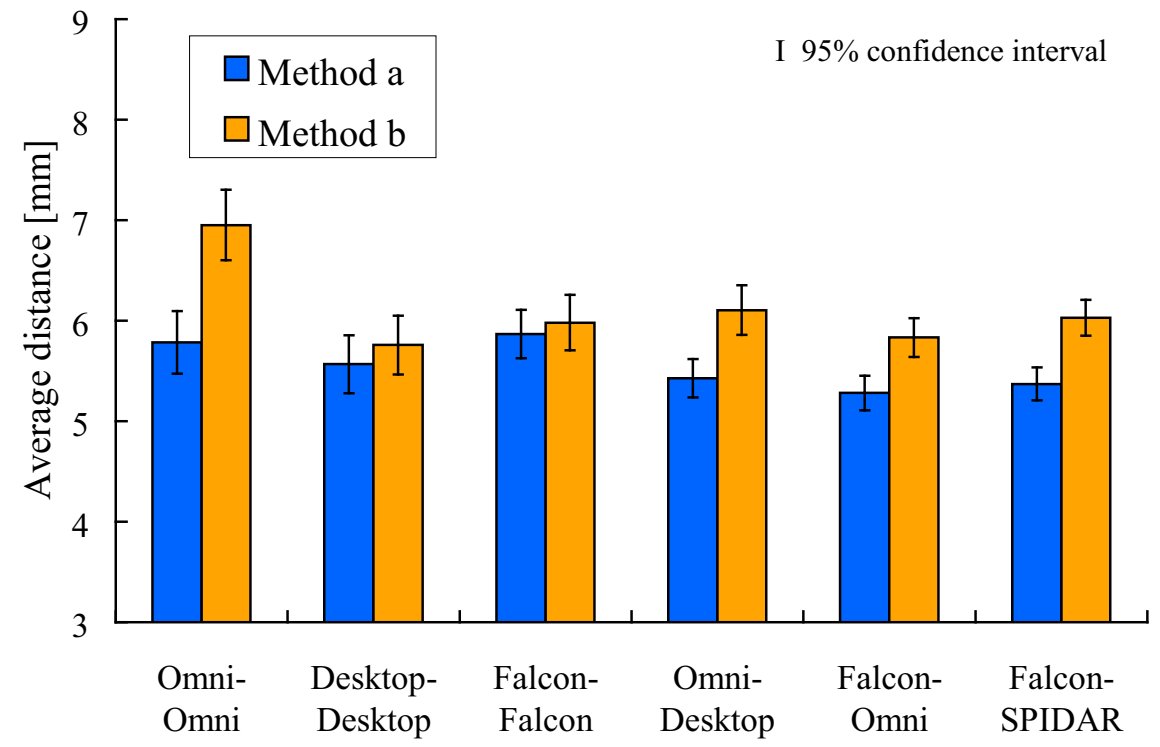

Fig. 10. Average distance between cube and target in case where virtual space size is set to reference size

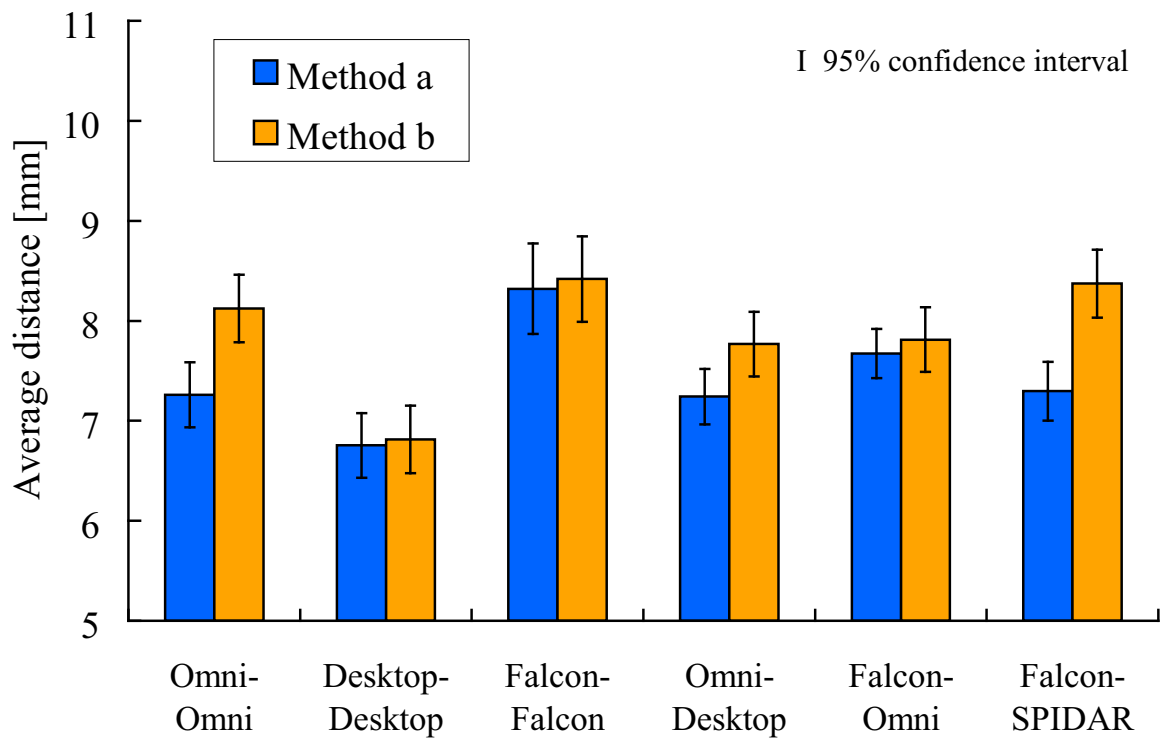

Fig. 11. Average distance between cube and target in case where virtual space size is set to one and a half times reference size 


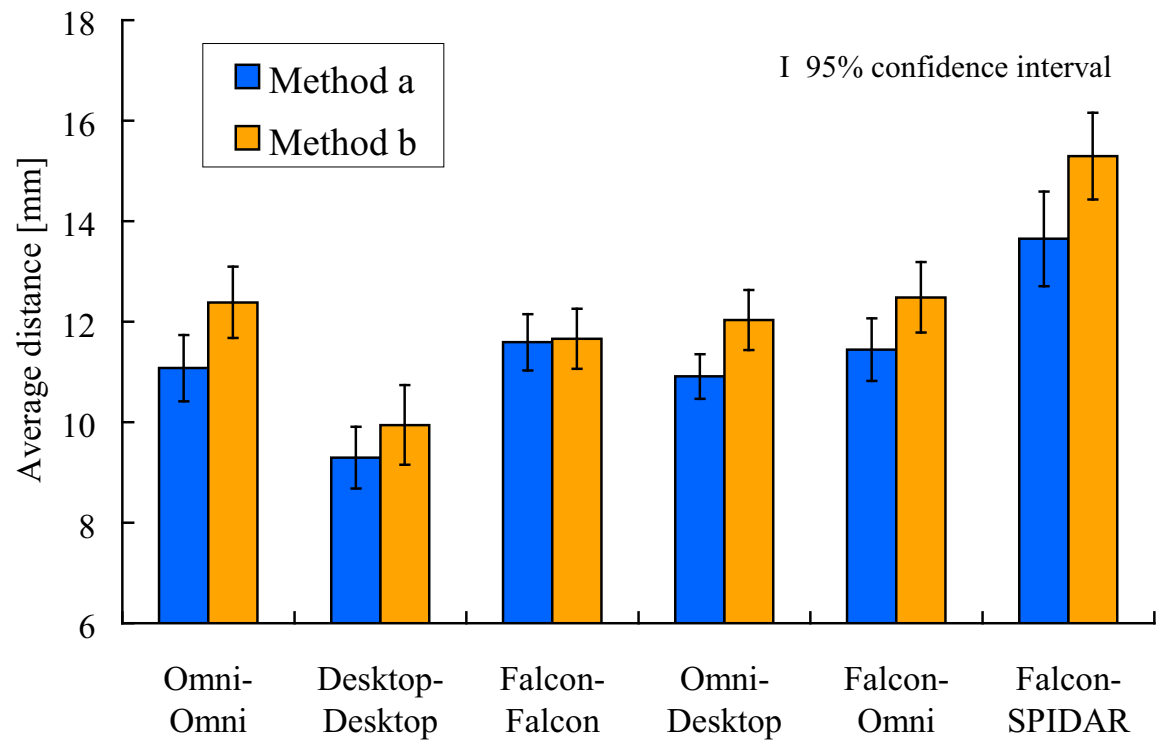

Fig. 12. Average distance between cube and target in case where virtual space size is set to twice reference size

From Figures 13, 14, and 16, we find that the average total number of eliminated targets of Method a is larger than that of Method $b$. The reason is similar to that in the case of the collaborative work. In Figure 15, the average total number of eliminated targets of Method b is somewhat larger than that of Method a. To clarify the reason, we examined the average number of eliminated targets at each haptic interface devices. As a result, the average number of eliminated targets of Omni with Method $b$ was larger than that with Method a. This is because in the case of Omni, the mapping ratio of the $x$-axis with Method a is much larger than that with Method b owing to the shape of the workspace of Omni; therefore, it is easy to drop the cube in Method a.

From the above observations, we can roughly conclude that Method a is more effective than Method $b$ in the competitive work.

\section{Conclusion}

This chapter dealt with collaborative work and competitive work using four kinds of haptic interface devices (Omni, Desktop, SPIDAR, and Falcon) when the size of a virtual space is different from the size of each workspace. We examined the influences of methods of mapping workspaces to the virtual space on the efficiency of work. As a result, we found that the efficiency of work is higher in the case where the workspace is uniformly mapped to the virtual space in the directions of the $x-, y$-, and $z$-axes than in the case where the workspace is individually mapped to the virtual space in the direction of each axis so that the mapped workspace size corresponds to the virtual space size. 


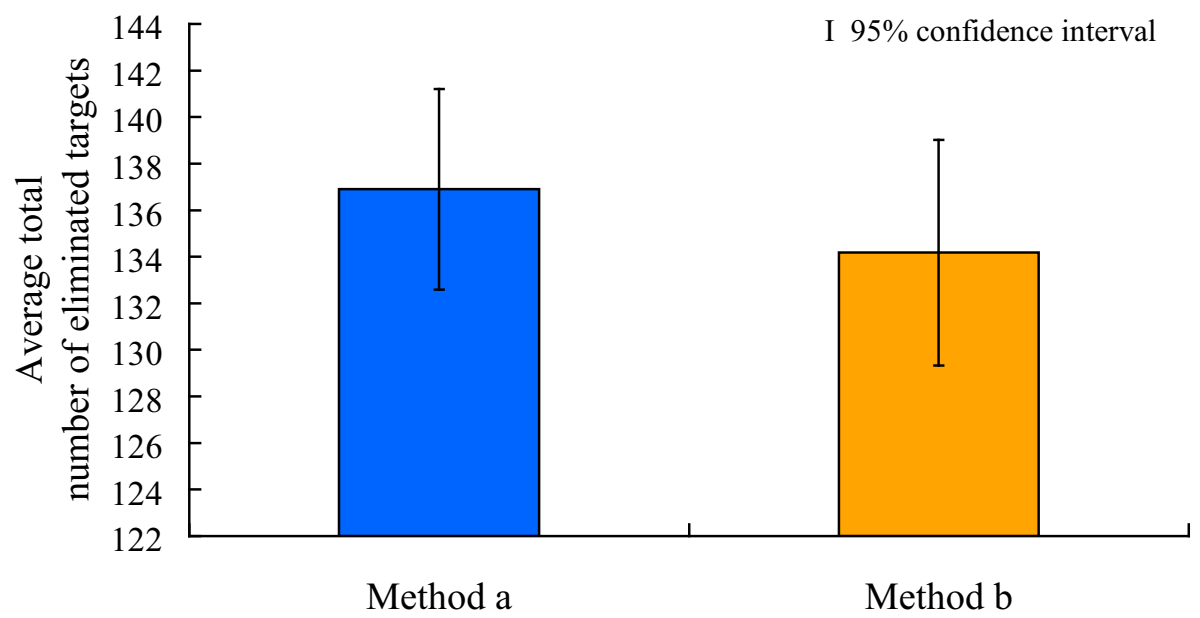

Fig. 13. Average total number of eliminated targets in case where virtual space size is set to half reference size

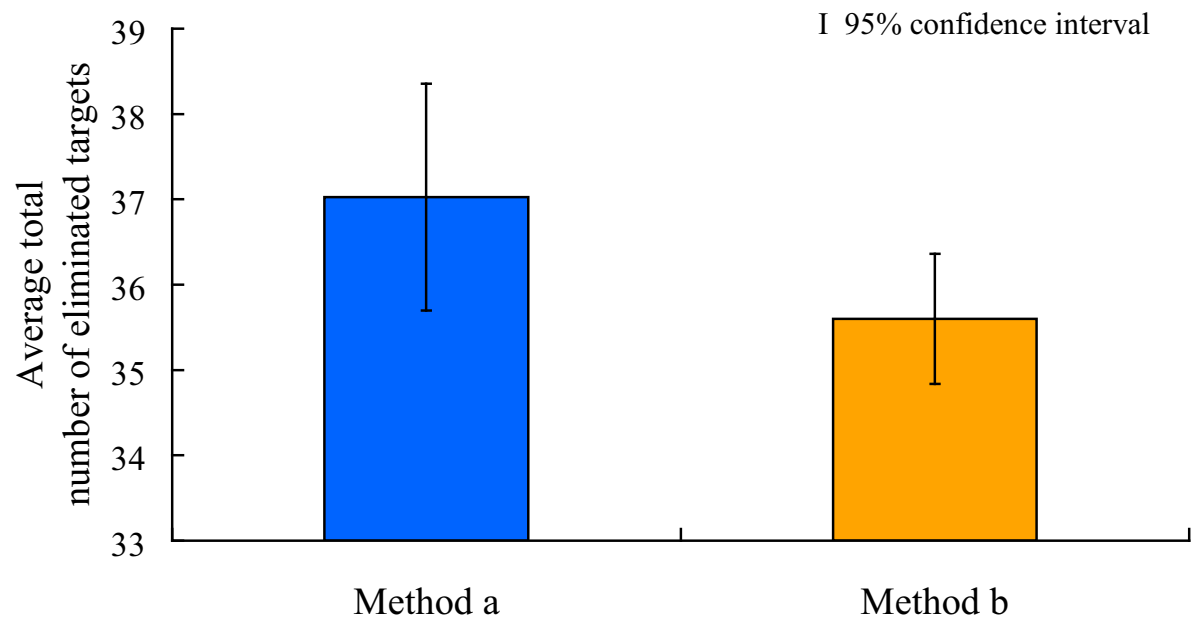

Fig. 14. Average total number of eliminated targets in case where virtual space size is set to reference size 


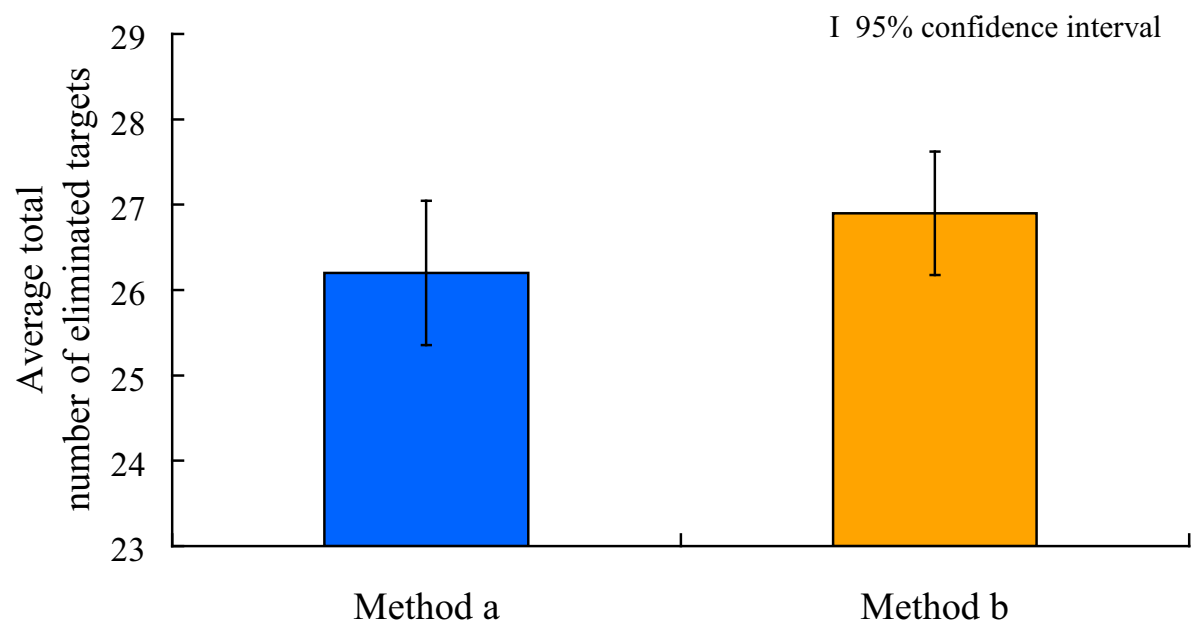

Fig. 15. Average total number of eliminated targets in case where virtual space size is set to one and a half times reference size

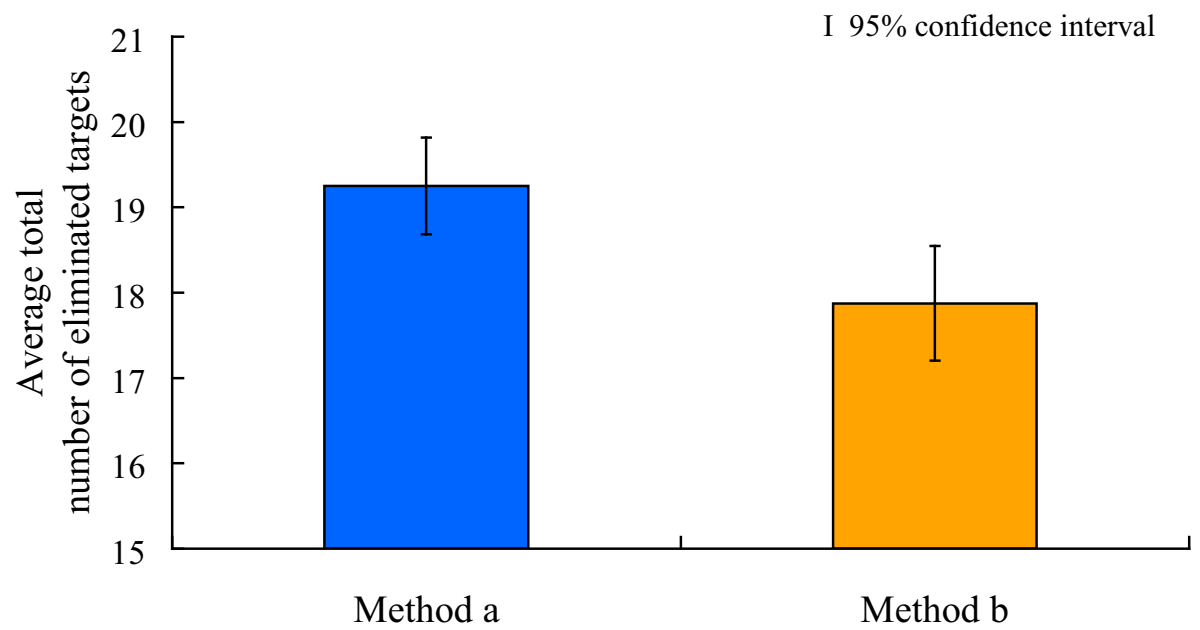

Fig. 16. Average total number of eliminated targets in case where virtual space size is set to twice reference size 
As the next step of our research, we will handle other types of work and investigate the influences of network latency and packet loss.

\section{Acknowledgments}

The authors thank Prof. Shinji Sugawara and Prof. Norishige Fukushima of Nagoya Institute of Technology for their valuable comments.

\section{References}

Fujimoto, T.; Huang, P.; Ishibashi, Y. \& Sugawara, S. (2008). Interconnection between different types of haptic interface devices: Absorption of difference in workspace size, Proceedings of the 18th International Conference on Artificial Reality and Telexistence (ICAT'08), pp. 319-322

Hirose, M.; Iwata, H.; Ikei, Y.; Ogi, T.; Hirota, K.; Yano, H. \& Kakehi, N. (1998). Development of haptic interface platform (HIP) (in Japanese). TVRSJ, Vol. 10, No. 3, pp. 111-119

Huang, P.; Fujimoto, T.; Ishibashi, Y. \& Sugawara, S. (2008). Collaborative work between heterogeneous haptic interface devices: Influence of network latency, Proceedings of the 18th International Conference on Artificial Reality and Telexistence (ICAT'08), pp. 293-296

Ishibashi, Y. \& Kaneoka, H. (2006). Group synchronization for haptic media in a networked real-time game. IEICE Trans. Commun., Special Section on Multimedia QoS Evaluation and Management Technologies, Vol. E89-B, No. 2, pp. 313-319

Ishibashi, Y.; Tasaka, S. \& Hasegawa, T. (2002). The virtual-time rendering algorithm for haptic media synchronization in networked virtual environments, Proceedings of the 16th International Workshop on Communication Quality \& Reliability (CQR'02), pp. 213217

Kameyama, S. \& Ishibashi, Y. (2007). Influences of difference in workspace size between haptic interface devices on networked collaborative and competitive work, Proceedings of SPIE Optics East, Multimedia Systems and Applications X, Vol. 6777, No. 30

Kim, S.; Berkley, J. J. \& Sato, M. (2003). A novel seven degree of freedom haptic device for engineering design. Virtual Reality, Vol. 6, No. 4, pp. 217-228

Novint Technologies, Inc. (2007). Haptic Device Abstraction Layer programmer's guide, Version 1.1.9 Beta

Salisbury, J. K. \& Srinivasan, M. A. (1997). Phantom-based haptic interaction with virtual object. IEEE Computer Graphics and Applications, Vol. 17, No. 5, pp. 6-10

SensAble Technologies, Inc. (2004). 3D Touch SDK OpenHaptics Toolkit programmer's guide, Version 1.0

Srinivasan, M. A. \& Basdogn, C. (1997). Haptics in virtual environments: Taxonomy, research status, and challenges. Computers and Graphics, Vol. 21, No. 4, pp. 393-404 


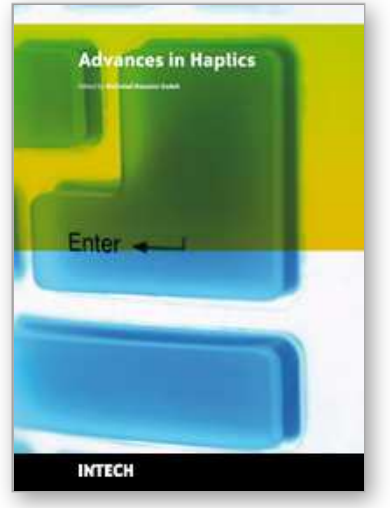

\author{
Advances in Haptics \\ Edited by Mehrdad Hosseini Zadeh
}

ISBN 978-953-307-093-3

Hard cover, 722 pages

Publisher InTech

Published online 01, April, 2010

Published in print edition April, 2010

Haptic interfaces are divided into two main categories: force feedback and tactile. Force feedback interfaces are used to explore and modify remote/virtual objects in three physical dimensions in applications including computer-aided design, computer-assisted surgery, and computer-aided assembly. Tactile interfaces deal with surface properties such as roughness, smoothness, and temperature. Haptic research is intrinsically multidisciplinary, incorporating computer science/engineering, control, robotics, psychophysics, and human motor control. By extending the scope of research in haptics, advances can be achieved in existing applications such as computer-aided design (CAD), tele-surgery, rehabilitation, scientific visualization, robot-assisted surgery, authentication, and graphical user interfaces (GUI), to name a few. Advances in Haptics presents a number of recent contributions to the field of haptics. Authors from around the world present the results of their research on various issues in the field of haptics.

\title{
How to reference
}

In order to correctly reference this scholarly work, feel free to copy and paste the following:

Ayano Tatematsu and Yutaka Ishibashi (2010). Mapping Workspaces to Virtual Space in Work Using Heterogeneous Haptic Interface Devices, Advances in Haptics, Mehrdad Hosseini Zadeh (Ed.), ISBN: 978953-307-093-3, InTech, Available from: http://www.intechopen.com/books/advances-in-haptics/mappingworkspaces-to-virtual-space-in-work-using-heterogeneous-haptic-interface-devices

\section{INTECH}

open science | open minds

\section{InTech Europe}

University Campus STeP Ri

Slavka Krautzeka 83/A

51000 Rijeka, Croatia

Phone: +385 (51) 770447

Fax: +385 (51) 686166

www.intechopen.com

\section{InTech China}

Unit 405, Office Block, Hotel Equatorial Shanghai No.65, Yan An Road (West), Shanghai, 200040, China 中国上海市延安西路65号上海国际贵都大饭店办公楼 405 单元 Phone: +86-21-62489820

Fax: $+86-21-62489821$ 
(C) 2010 The Author(s). Licensee IntechOpen. This chapter is distributed under the terms of the Creative Commons Attribution-NonCommercialShareAlike-3.0 License, which permits use, distribution and reproduction for non-commercial purposes, provided the original is properly cited and derivative works building on this content are distributed under the same license. 